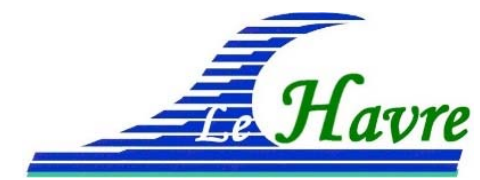

\title{
Réflexion sur la prise en considération du recul du trait de côte dans la conception de l'ouvrage de protection côtière de Lacanau Océan
}

\author{
Arnaud SALLABERRY ${ }^{1}$, Jérémy DUGOR ${ }^{1}$, Julien BAILLS ${ }^{1}$, Didier RIHOUEY ${ }^{1}$ \\ 1. CASAGEC INGENIERIE, 18 rue Maryse Bastié, Zone de Maignon, 64600 Anglet, \\ France. \\ contact@casagec.fr
}

\section{Résumé :}

Le front de mer de Lacanau Océan, comme une grande partie du littoral français, est confronté à la problématique du recul du trait de côte. Ainsi, un ouvrage de fixation du trait de côte a été installé à partir des années 80 pour protéger les enjeux les plus vulnérables, situés de plus en plus proche du rivage. Cette protection a été reprise en urgence après avoir été partiellement détruite à la suite des évènements tempétueux de l'hiver 2013-2014, saison la plus énergétique observée depuis près de 60 ans (MASSELINK et al., 2016). Durant ces tempêtes, un fort recul du trait de côte (CASTELLE et al., 2015) et un abaissement généralisé de l'estran face à l'ouvrage ont été observés provoquant la déstabilisation de l'ouvrage. Afin de pérenniser à moyen terme cet ouvrage réalisé dans l'urgence, une réflexion a été mise en place permettant de prendre en considération le recul du trait de côte.

Dans la zone d'implantation des ouvrages de Lacanau, les fonds marins et les plages, étant donné leurs compositions sableuses et les sollicitations hydrodynamiques, sont mobiles et évolutifs. Ces évolutions se produisent à plusieurs échelles temporelles. Des évolutions à court terme sont observées en lien avec une tempête, un cycle de marée et les saisons. Mais des évolutions à plus long terme associées au recul moyen du trait de côte sont également à prendre en considération.

Afin de caractériser ces évolutions à court terme, un travail d'analyse a été réalisé sur des levés topographiques de la plage couvrant la période 2003 - 2018. 13 profils localisés le long des 1,2 km d'ouvrage de protection du front de mer de Lacanau ont été comparés permettant de définir des profils théoriques considérés défavorables au regard de la conception des ouvrages à court terme.

La réflexion concernant l'évolution des profils de plage à long terme s'appuie sur la projection du trait de côte à horizon 2050, aux extrémités Nord et Sud de la protection côtière, réalisée par l'Observatoire de la Côte Aquitaine (BERNON et al., 2016). De plus, au niveau d'un ouvrage fixant le trait de côte, le recul se traduit par un abaissement des fonds, tel qu'évoqué dans le Guide Méthodologique du Plan de prévention des risques littoraux (MEDDE, 2014). 


\section{Thème 7 - Risques côtiers}

Dans le cas de Lacanau, deux hypothèses d'abaissement sont proposées et les conséquences associées sur la conception de l'ouvrage sont présentées. Il en ressort que les conséquences du recul du trait de côte sont majeures et doivent impérativement être prises en considération lors du dimensionnement de l'ouvrage.

Mots-clés :

Conception, Ouvrages côtiers, Evolutions des profils de plage, Recul du trait de côte.

\section{Introduction}

Selon l'indicateur national de l'érosion côtière (HÉDOU et al., 2018) 19\% des côtes maritimes française doivent faire face à une problématique d'érosion et ce pourcentage atteint $37 \%$ pour les côtes sableuses. En réponse à cette problématique de recul du trait de côte et au problème de submersion, certains fronts de mer ont fait l'objet d'artificialisation à travers l'édification d'ouvrages de protection. Depuis les années 60, une forte augmentation du linéaire artérialisé a été observé atteignant 30\% du trait de côte. La réalisation d'un ouvrage de protection permet généralement de n'apporter qu'une réponse ponctuelle géographiquement et temporellement aux problématiques d'érosion et de recul du trait de côte. En effet, même si la présence de l'ouvrage permet de figer le trait de côte, la problématique d'érosion n'en est pas pour autant annulée et cette érosion se traduit notamment par un abaissement du niveau de sable devant l'ouvrage et un décalage aux extrémités de l'ouvrage en question. Le Guide Méthodologique du Plan de prévention des risques littoraux (MEDDE, 2014) évoque le cas d'un ouvrage permettant de figer temporaire le trait de côte : "Dans ce cas, la zone soumise à aléa est déterminée par rapport à l'évolution des zones naturelles non protégées adjacentes où la dynamique érosive demeure active. En effet, le recul des zones contiguës à la zone protégée conduira à un contournement de l'ouvrage de protection. L'ouvrage procure ainsi une protection pendant une durée limitée dans le temps, la protection devenant de plus en plus difficile à assurer au fur et à mesure du recul des zones adjacentes."

Dans le cas de Lacanau, l'ouvrage édifié à partir des années 80 permet de fixer localement le front de mer qui se trouve ainsi en avant du trait de côte naturel (figure 1).

L'influence du recul du trait de côte sur la conception d'un ouvrage n'est que rarement prise en considération dans les projets et aucune méthodologie n'est clairement explicitée dans la littérature. Il est cependant indispensable, au même titre que l'effet du changement climatique, de prendre en considération le recul du trait de côte dès la phase de conception. De ce fait, dans un contexte érosif, la conception d'un nouvel ouvrage ou la vérification de la stabilité d'un ouvrage existant nécessite d'estimer une valeur d'abaissement en pied d'ouvrage. Cet abaissement aura un effet direct sur la stabilité de l'ouvrage (ancrage, grand glissement...) mais également sur les houles atteignant l'ouvrage pouvant ainsi influencer la stabilité de la carapace, les volumes de franchissements... 


\section{XVİ̀mes Journées Nationales Génie Côtier - Génie Civil \\ Le Havre 2020}

L'objet du présent article consiste à présenter l'approche mise en œuvre afin de déterminer une valeur d'abaissement en pied de l'ouvrage de Lacanau Océan en vue d'étudier la pérennisation de ce dernier jusqu'à un horizon 2050. Cette date de 2050 a été retenue pour permettre à la commune d'envisager le devenir de son front de mer.

\section{Présentation du site d'étude, du contexte érosif et de l'ouvrage}

Lacanau Océan constitue le front de mer de la commune de Lacanau qui est située dans le département de la Gironde. Lacanau Océan se trouve dans une zone où la côte est sableuse et en recul comme la quasi-totalité des côtes Girondines. La marée y est de type semi-diurne avec un marnage astronomique d'amplitude $5,30 \mathrm{~m}$ avec une valeur pour la PHMA de 5,30 m CM ou 2,92 m IGN 69 (SHOM, 2019). Les houles d'Atlantique de provenance Ouest ont une hauteur significative moyenne au large de $1,9 \mathrm{~m}$, cette valeur atteint $14,5 \mathrm{~m}$ pour un événement extrême ayant une période de retour de 100 ans.

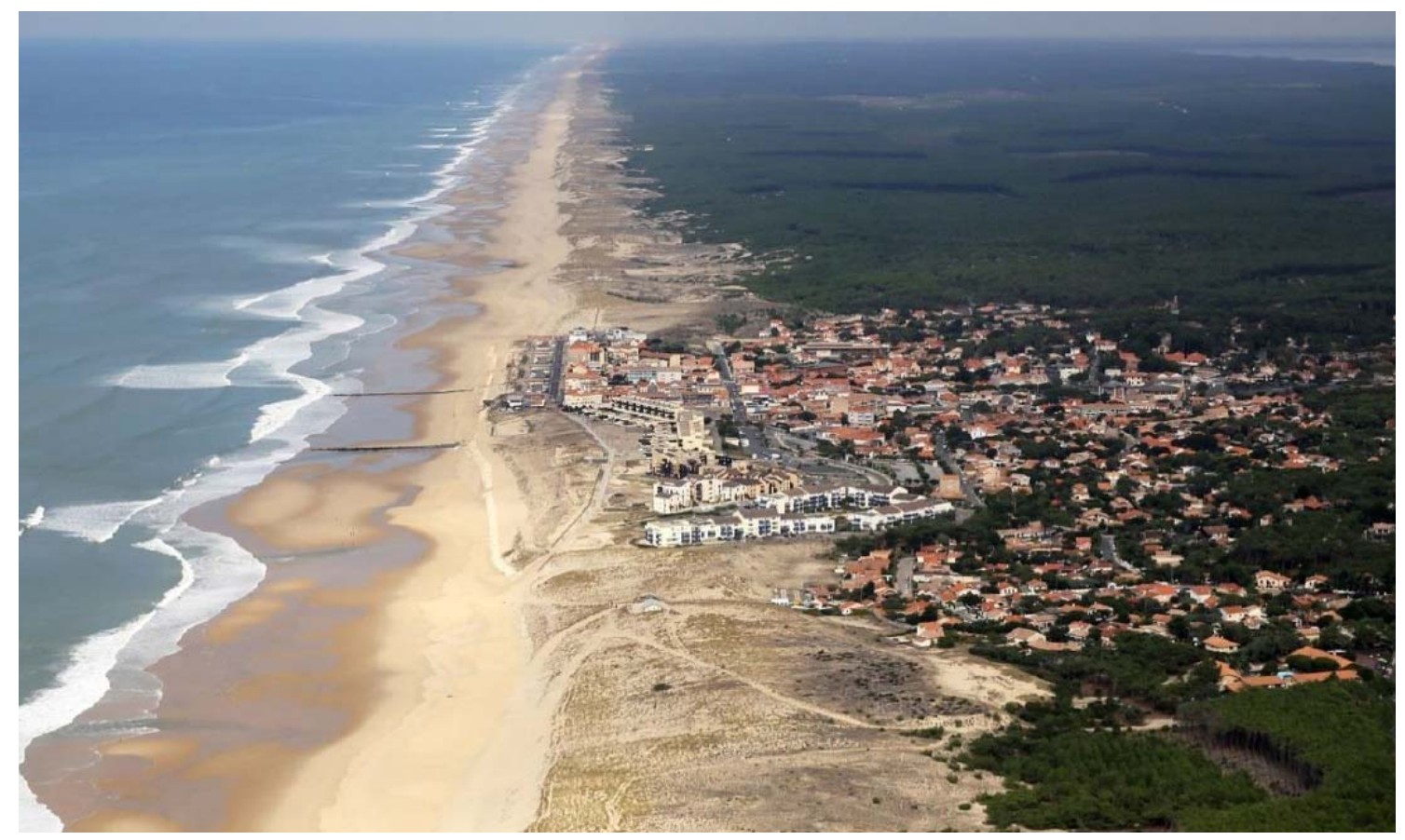

Figure 1. Photographie aérienne de Lacanau Océan.

Le travail produit par l'Observatoire de la Côte Aquitaine (OCA-BRGM, 2016) permet de définir des projections du trait de côte à horizons 2025 et 2050 au Nord et au Sud de la zone urbanisée et artificialisée de Lacanau Océan. Ces projections sont définies à partir de l'estimation d'un recul moyen historique ( 9 traits de côte historiques entre 1985 et 2014), de l'estimation d'un recul lié à un évènement majeur ( $\mathrm{L} \max =25 \mathrm{~m}$ ) et de l'estimation d'un recul lié au changement climatique $(20 \mathrm{~m})$. On notera que l'ensemble de la côte sableuse en Gironde est en érosion avec des taux compris entre $0,5 \mathrm{~m} / \mathrm{an}$ et $14,7 \mathrm{~m} / \mathrm{an}$. 


\section{Thème 7 - Risques côtiers}

La définition de l'abaissement du sable en pied d'ouvrage sera réalisée en considérant à horizon 2050 un recul du trait de côte de $77 \mathrm{~m}$ au Nord et $82 \mathrm{~m}$ au Sud soit de $80 \mathrm{~m}$ en moyenne dans la zone étudiée.

Sur 1,1 km de front de mer, Lacanau Océan est protégé par un ouvrage en enrochements directement adossé à la dune de sable, la ville se trouvant en crête ou en arrière immédiat de cette dune (figure 2).

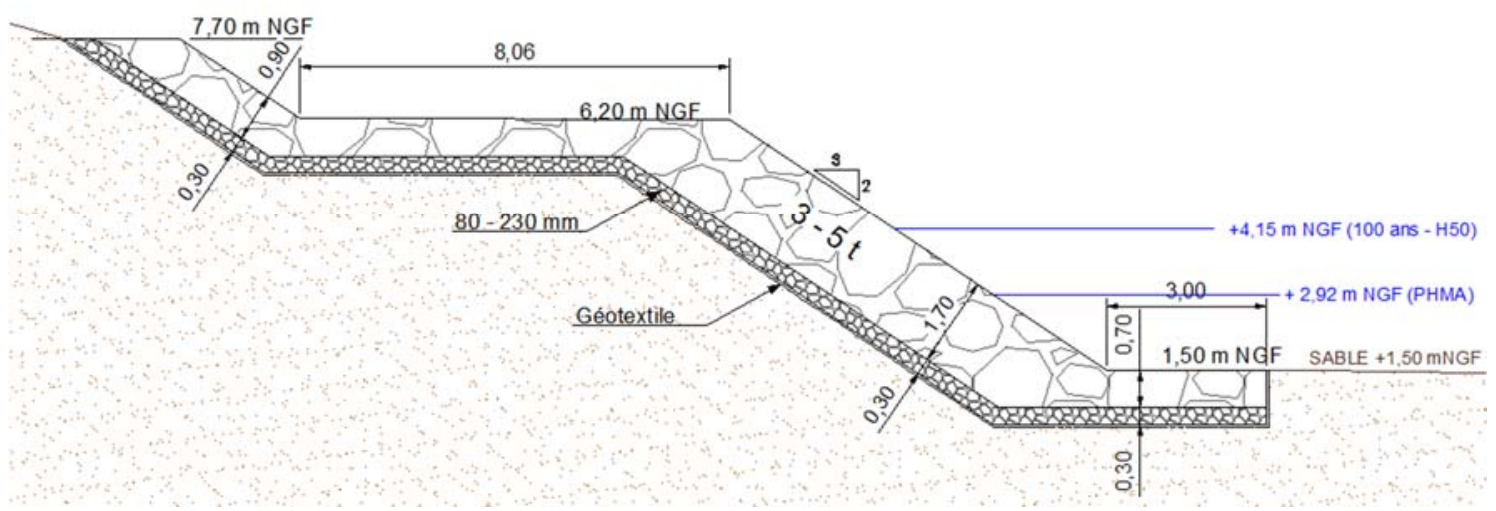

Figure 2. Coupe-type de l'ouvrage actuel dans sa configuration berme.

\section{Estimation de l'abaissement de l'estran}

\subsection{Introduction}

Entre 2003 et 2016 des levés de profils topographiques de la plage ont été réalisés. Depuis 2016, un observatoire du littoral a été mis en place dans le cadre de la stratégie locale de gestion du trait de côte de Lacanau, comprenant un levé topo-bathymétrique de l'ensemble de la zone ouvrage et cordon dunaire et cela plusieurs fois par an. Ces données ont été analysées afin d'essayer de comprendre le fonctionnement des fonds devant l'ouvrage.

\subsection{Evolution du stock sédimentaire}

Les levés topo-bathymétriques (2016-2018) permettent de visualiser l'évolution de la plage et de faire ressortir, à travers la création de cartes de comparaison, les zones d'accrétion et les zones d'érosion (figure 3). De plus, les volumes associés à ces accrétions et ces érosions peuvent être estimés pour la zone urbanisée (centre) ainsi que pour les zones adjacentes Nord et Sud, tableau 1. 


\section{XVI èmes Journées Nationales Génie Côtier - Génie Civil \\ Le Havre 2020}
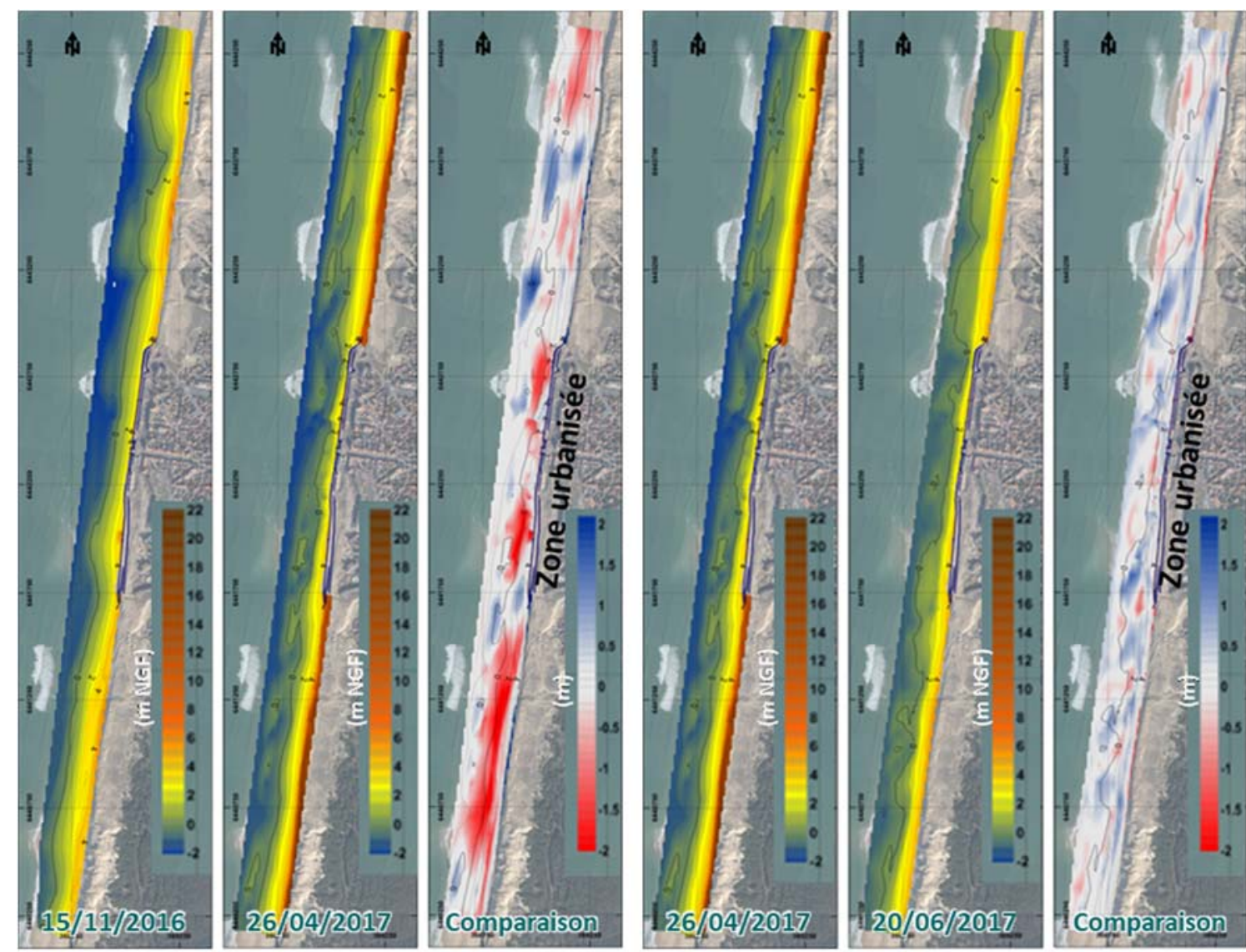

Figure 3. Exemple de cartes des levés topographiques et des comparaisons.

Tableau 1. Volumes d'érosion et d'accrétion pour plusieurs périodes et trois zones.

\begin{tabular}{|c|c|c|c|r|r|r|}
\hline Date 1 & Date 2 & Durée (j) & Zone & Erosion $\left.\boldsymbol{( m}^{3}\right)$ & Accrétion $\left(\boldsymbol{m}^{3}\right)$ & Volume net $\left(\mathbf{m}^{3}\right)$ \\
\hline \multirow{3}{*}{$15 / 11 / 2016$} & \multirow{3}{*}{$20 / 04 / 2017$} & \multirow{3}{*}{162} & Nord & -79600 & 110000 & 30400 \\
\cline { 4 - 7 } & & Centre & -92500 & 42200 & -50300 \\
\cline { 4 - 7 } & & Sud & -194800 & 53800 & -141000 \\
\hline \multirow{3}{*}{$26 / 04 / 2017$} & \multirow{3}{*}{$20 / 06 / 2017$} & \multirow{2}{*}{55} & Nord & -49900 & 78500 & 28600 \\
\cline { 4 - 7 } & & Centre & -23700 & 72600 & 48900 \\
\cline { 4 - 7 } & & Sud & -33000 & 79200 & 46200 \\
\hline \multirow{3}{*}{$20 / 06 / 2017$} & \multirow{3}{*}{$20 / 10 / 2017$} & \multirow{2}{*}{122} & Nord & -44000 & 107800 & 63800 \\
\cline { 4 - 7 } & & Centre & -12400 & 87500 & 75100 \\
\cline { 4 - 7 } & & Sud & -22800 & 93600 & 71800 \\
\hline \multirow{3}{*}{$20 / 10 / 2017$} & \multirow{3}{*}{$20 / 03 / 2018$} & \multirow{2}{*}{151} & Nord & -271600 & 24900 & -246700 \\
\cline { 4 - 7 } & & & Centre & -170400 & 12300 & -158100 \\
\cline { 4 - 7 } & & Sud & -169900 & 46500 & -123400 \\
\hline
\end{tabular}

Ainsi, l'analyse des levés topo-bathymétriques permet de faire ressortir de fortes évolutions des volumes de sable au droit des ouvrages avec de fortes disparités géographiques et temporelles. L'ordre de grandeur de l'évolution du volume net sur un mois est de $100000 \mathrm{~m}^{3}$ pour l'ensemble de la zone et de $30000 \mathrm{~m}^{3}$ au droit de la protection côtière. Même si la période de mesure est relativement faible, une saisonnalité 


\section{Thème 7 - Risques côtiers}

est observée avec une période estivale d'accrétion et une période hivernale d'érosion malgré une tendance moyenne à l'érosion. Il est fort probable qu'une partie des échanges saisonniers se fassent entre la plage et les petits fonds/ barres sableuses. De plus, les rechargements annuels en sable de $5000 \mathrm{~m}^{3}$ réalisés au niveau de la protection côtière n'ont qu'une influence très limitée sur le fonctionnement hydro-sédimentaire global du site.

3.3 Evolution des profils de plage et définition de profils théoriques enveloppes

Sur une période comprise entre 2003 et 2018 de nombreux profils topographiques de plage ont été réalisés pour 11 dates le long du front de mer de Lacanau Océan (figure 4).
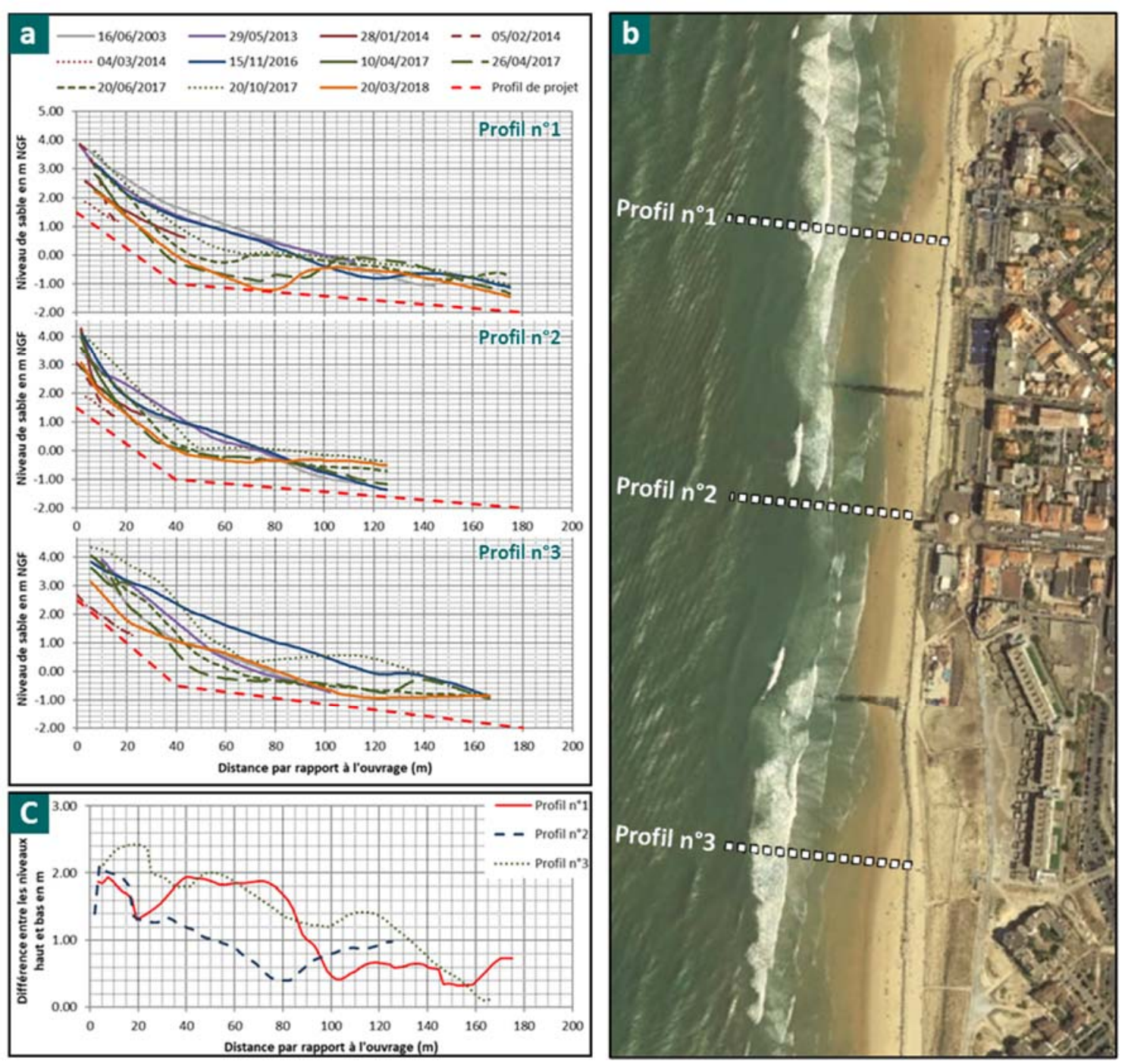

Figure 4. a. Profils topographiques / b. localisation des profils / c. variation topographique des profils. 


\section{XVİ̀mes Journées Nationales Génie Côtier - Génie Civil \\ Le Havre 2020}

Trois profils topographiques et leurs variations (valeur maximum - minimum) réalisés au droit de la protection côtière sont présentés dans la figure 4. L'analyse de ces levés topographiques représentatifs permet de montrer la forte variabilité des profils de plage. La variabilité observée est de l'ordre de 1 à $2 \mathrm{~m}$ avec une plus forte valeur à proximité de l'ouvrage.

Malgré le nombre de levés relativement élevé, l'information concernant l'évolution des fonds devant l'ouvrage reste partielle car elle ne représente que 11 dates sur 15 années et ne permet pas de représenter la configuration des fonds lors d'une tempête.

\subsection{Hypothèses, choix et influence sur la conception de l'abaissement des fonds}

Les conséquences du recul du trait de côte sur l'ouvrage se traduisent par deux phénomènes le contournement de ce dernier au Nord et au Sud et un abaissement en pied d'ouvrage. Le contournement de l'ouvrage est traité par une disposition ponctuelle consistant à réaliser des retours d'ouvrage qui s'ancrent dans la dune. L'effet de l'abaissement est pris en considération par une approche détaillée ci-dessous.

Afin de définir une configuration de "conception" permettant de réaliser les calculs indispensables au dimensionnement ou à la vérification de l'ouvrage, 2 hypothèses concernant le profil de plage ont été réalisées.

La première hypothèse consiste à retenir 2 profils de plage théoriques appelés "profils de projet" et présentés par l'intermédiaire d'une ligne discontinue rouge dans la figure 4. Ces profils de projet constituent l'enveloppe basse, de l'ensemble des profils mesurés le long des ouvrages et cela pour les 11 dates. Il est fort probable que des profils, localement et à un instant $t$, puissent être plus bas que ces valeurs empiriques. Cependant, dans le cas d'un ouvrage linéaire comme celui de Lacanau les phénomènes hydrauliques (propagation de la houle, franchissement, efforts sur l'ouvrage...) peuvent s'équilibrer et de ce fait être moyennés sur un certain linéaire d'ouvrage. De plus, la concomitance d'un niveau de plage extrêmement bas avec le pic d'une tempête exceptionnelle a une faible probabilité et est très dépendante de la configuration de la plage avant la tempête. De ce fait, cette première hypothèse est considérée comme offrant une marge de sécurité suffisante sans pour autant amener au surdimensionnement de l'ouvrage.

La seconde hypothèse concerne la prise en considération du recul du trait de côte dans l'abaissement des fonds devant l'ouvrage. La valeur moyenne de recul dans le périmètre du front de mer de Lacanau Océan est de l'ordre de $80 \mathrm{~m}$ à horizon 2050. Au niveau des ouvrages ce recul se traduit par un abaissement. 2 options sont envisageables pour traduire cet abaissement, figure 5 :

- Option 1 - la forme du profil de plage devant l'ouvrage s'adapte et se maintient et un abaissement de $80 \mathrm{~cm}$ est appliqué en accord avec la pente moyenne des fonds de 1\% dans la zone d'étude ;

- Option 2 - le profil est purement translaté horizontalement de $80 \mathrm{~m}$ ce qui implique un abaissement brutal compris entre 2,9 et 3,9 m. En effet, dans le cas d'une 


\section{Thème 7 - Risques côtiers}

translation du profil, la remontée sableuse aujourd'hui observée devant l'ouvrage disparait.

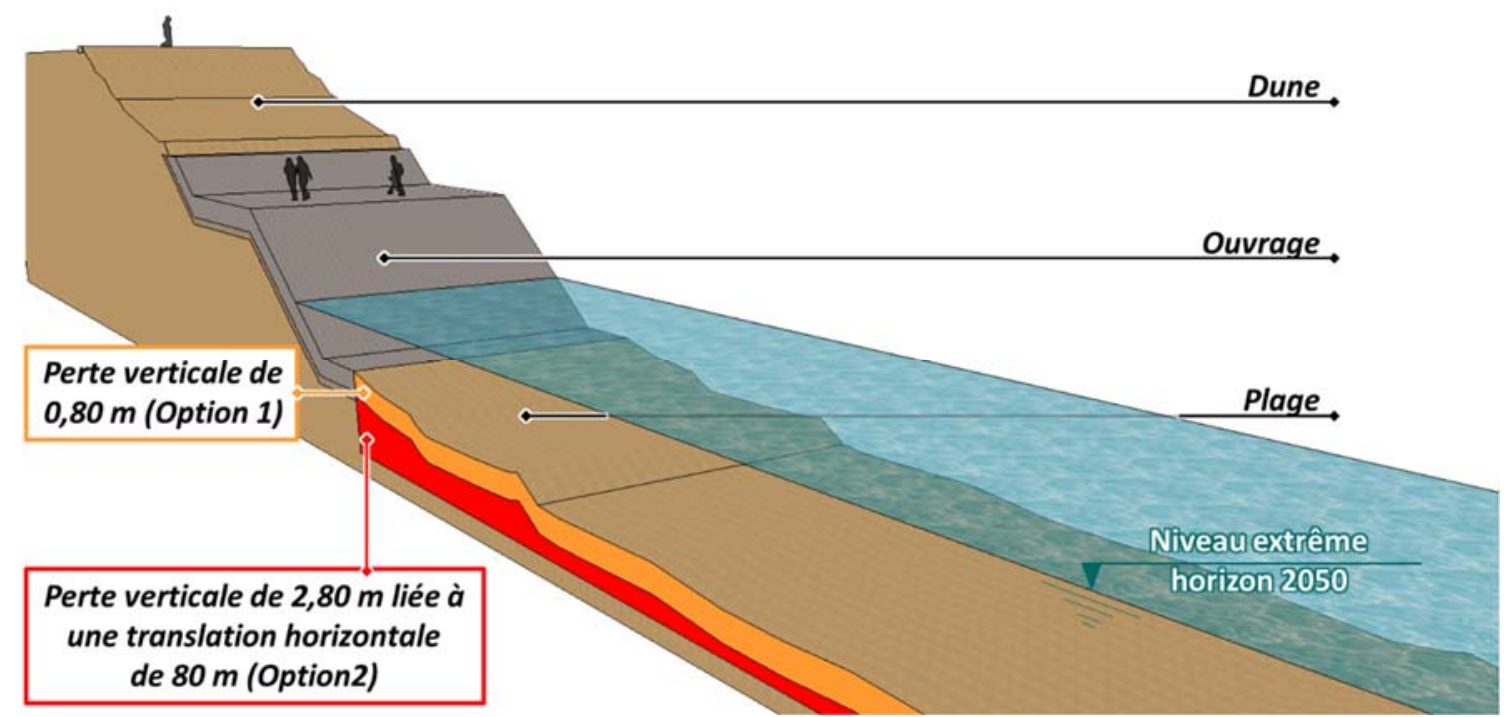

Figure 5. Illustration des 2 options concernant l'abaissement à horizon 2050.

L'option $\mathrm{n}^{\circ} 2$ a été retenue pour la définition d'un ouvrage à horizon 2050. Cette option est jugée la plus probable et elle ne se base pas sur le maintien hypothétique de la remontée sableuse existante aujourd'hui devant l'ouvrage.

En effet, l'option 2 apparait comme étant du côté de la sécurité, car étant plus contraignante vis-à-vis des risques d'affouillement et des houles de projet susceptibles d'atteindre l'ouvrage. Le choix a des conséquences très fortes sur la conception de l'ouvrage. L'ouvrage actuel n'est pas en mesure de résister à un tel abaissement, le futur ouvrage nécessiterait d'être fondé à -3,40 m IGN69 contre +0,5 m IGN69 aujourd'hui.

De plus, la hauteur de la houle susceptible d'atteindre l'ouvrage sera beaucoup plus importante avec une augmentation de l'ordre de $+30 \%$ pour l'option 1 et de $+100 \%$ pour l'option 2. En comparaison, sur la même période, la prise en considération de l'effet du changement climatique sur l'élévation du niveau moyen engendre une augmentation de l'ordre de $15 \%$ de la hauteur de la houle en pied d'ouvrage.

De ce fait, les conséquences sur le dimensionnement de l'ouvrage (niveau de crête, taille des éléments de carapace,...) sont très fortes. Ainsi, l'emprise, les quantités de matériaux et le coût du projet seront largement augmentés. Il ressort que dans le cas de l'option 2 la conception amène à un volume de matériaux nécessaire 4, 0 à 5,5 fois supérieur au volume de matériaux en place aujourd'hui.

\section{Conclusion et discussion}

Le recul du trait de côte est aujourd'hui trop peu pris en considération dans la conception ou la vérification des ouvrages côtiers. Or, il ressort que son influence sur la conception 


\section{XVI'̀mes Journées Nationales Génie Côtier - Génie Civil \\ Le Havre 2020}

des ouvrages peut-être du même ordre de grandeur voir bien supérieur à l'effet du changement climatique et cela même à faible échéance. Cependant, la complexité de l'évolution des fonds et des plages sableuses rend difficile la prise en considération de la géométrie des fonds et de leur possible évolution dans la conception des ouvrages côtiers. En effet, malgré le grand nombre de données topographiques et bathymétriques disponibles au niveau du site de Lacanau Océan, il est très difficile de caractériser les fonds, leur évolution à court et à long termes au droit de l'ouvrage. Devant cette complexité, l'approche retenue dans le cadre de Lacanau a consisté à simplifier de manière significative la problématique, en travaillant à partir de profils théoriques. Ces profils ont été abaissés selon 2 options d'abaissement (option 1, translation verticale et option 2, translation horizontale du profil de plage). Ces options 1 et 2 engendrent respectivement une augmentation de la hauteur de houle en pied d'ouvrage de 30 et de $100 \%$. Sur la base d'échanges avec les différents acteurs du projet, l'option 2 a été retenue pour la conception de l'ouvrage car à la fois réaliste et plus défavorable. Le travail réalisé a ainsi permis d'appréhender l'impact significatif de l'abaissement des fonds sur la vérification et ou la conception d'un ouvrage de protection côtière.

Toutefois, cette approche simplifiée conduisant à la définition de plusieurs hypothèses questionne sur la légitimité de ces dernières et met en lumière un certain nombre de sujets à approfondir. Continuer à améliorer la connaissance du fonctionnement hydrosédimentaire du site afin d'essayer de caractériser de manière plus précise et plus réaliste son évolution à venir apparait comme une piste d'optimisation.

De plus, malgré le caractère linéaire du trait de côte sableux et des ouvrages de protection associés, les phénomènes hydro-sédimentaires devant les ouvrages sont tridimensionnels et il serait idéalement souhaitable de prendre en considération cette réalité 3D.

\section{Références bibliographiques}

CASTEllE B., MARIEU V., BUJANA S., SPLINTER K.D., ROBINET A., SENECHAL N., FERREIRA S. (2015). Impact of the winter 2013-2014 series of severe Western Europe storms on a double-barred sandy coast: Beach and dune erosion and megacusp embayments, Geomorphology, Vol. 238, pp 135-148. https://doi.org/10.1016/j.geomorph.2015.03.006

HÉDOU F., ROCHE A., TRMAL C., MORAUD S., DENIAU Y. (2018). Élaboration de l'indicateur national de l'érosion côtière, $\mathrm{XV}^{\mathrm{ème}}$ Journées Nationales Génie Côtier Génie Civil, La Rochelle, pp 647-654. https://doi.org/10.5150/jngcgc.2018.075

MASSELINK G., CASTELlE B., SCOTT T., DODOET G., SUANEZ S., JACKSON D., FLOC'H F. (2016). Extreme wave activity during 2013/2014 winter and morphological impacts along the Atlantic coast of Europe, Geophysical Research Letters, Volume 43, Issue 5. https://doi.org/10.1002/2015GL067492

MEDDE -Ministère de l'Écologie, du Développement durable, et de l'Energie- (2014). Guide méthodologique : Plan de prévention des risques littoraux, 169 p. 


\section{Thème 7 - Risques côtiers}

OCA BRGM -BERNON N., MALLET C., BELON R., HOAREAU A., BULTEAU T., GARNIER C.- (2016) Caractérisation de l'aléa recul du trait de côte sur le littoral de la côte aquitaine aux horizons 2025 et 2050, Rapport final, BRGM/RP-66277-FR, 200 p. SHOM (2019). Références Altimétriques Maritimes - Ports de France métropolitaine et d'outre-mer, Côtes du zéro hydrographique et niveaux caractéristiques de la marée. 